\title{
Performance analysis of a hybrid one-sided magnetic exciter mounted on a piezoelectric stack
}

\author{
A. Nandi* and S. Neogy \\ Department of Mechanical Engineering, Jadavpur University, Kolkata 700 032, India
}

Received 27 August 2008

Revised 2 June 2009

\begin{abstract}
The present work proposes a non-contact hybrid exciter especially useful for harmonic excitation of lightly damped structures/rotors. In the proposed exciter an electromagnet is placed on a piezoelectric stack and the extension of the piezoelectric stack is made almost equal to the displacement of the structure using a simple tracking control. This largely eliminates stiffness coupling between the structure/rotor and the exciter and non-linearity in the excitation force due to the vibration of the structure/rotor. The stiffness and inertia of the piezoelectric stack is considered in the analysis. A SIMULINK model of the combined structure and the exciter is developed for a full time-domain simulation of the excitation system.
\end{abstract}

Keywords: Piezoelectric stack, magnetic actuator, vibration exciter, non-contact excitation

\section{List of symbols}

$\begin{array}{ll}F & \text { Force exerted on structure by magnetic actuator } \\ k^{\prime} & \text { A constant, which depends on geometry, number of turns etc. of the electromagnet } \\ x_{0} & \text { The initial gap between the magnet and the structure/rotor } \\ x & \text { Displacement of the vibrating structure/rotor } \\ i & \text { Current in the coil of the electromagnet } \\ I & \text { Amplitude of harmonic current in the electromagnet } \\ \omega & \text { Frequency of harmonic current in the electromagnet } \\ x_{s 0}, \Delta x_{s} & \text { Mean position and increase in length of piezoelectric stack from mean position respectively } \\ \xi & \text { Length measured along piezoelectric stack } \\ x^{e} & \text { Displacement along the length of the piezoelectric stack } \\ x_{s} & \text { Displacement at the free end of the piezoelectric stack } \\ U & \text { Potential energy of the piezoelectric stack } \\ T & \text { Kinetic energy of the piezoelectric stack } \\ \bar{F} & \text { Force on the piezoelectric stack applied at the free end } \\ \sigma, \varepsilon & \text { Axial stress and strain in piezoelectric stack respectively } \\ E & \text { Young's modulus for the material of the piezoelectric stack } \\ d_{33} & \text { Piezoelectric constant } \\ \bar{l}, n & \text { Thickness of a single layer of the stack and number of such layers respectively } \\ A & \text { Cross-sectional area of the piezoelectric stack } \\ V_{0}, \Delta V & \text { Mean voltage and variation over mean voltage applied to the stack respectively } \\ \omega_{n s} & \text { Short circuited natural frequency of a stack with a magnet on it }\end{array}$

${ }^{*}$ Corresponding author. E-mail: arghyan@yahoo.com, anandi@mech.jdvu.ac.in. 


\section{Introduction}

Magnetic excitation of structure/rotor is attractive as excitation is possible without any physical contact [1,2]. For a rotor, it is very difficult to use conventional electrodynamic and modal excitation systems.

Sodano [3] has used eddy currents in a conducting plate for non-contact excitation of a beam. A permanent magnet is attached to a standard electrodynamic exciter. The gap between the magnet and the structure changes as the electrodynamic exciter vibrates. The changing magnetic field produces eddy current in a conducting plate. Due to the interaction between the magnetic fields produced by the permanent magnet and the eddy currents, the structure starts vibrating. However, since the forces due to eddy currents are generally small the application of such exciters is only limited to light structures.

In conventional magnetic actuator configuration used in active magnetic bearings (AMB), generally two counteracting electromagnets are used to apply force in a direction. Though this configuration largely reduces the inherent non-linearity of such actuators, it is difficult to place counter-acting pairs of magnets around a structure/rotor already in operation due to construction problems. Although the conventional magnetic actuator configuration can be used for the purpose of non-contact vibration excitation yet for a structure/rotor already in operation, it is much more convenient if the exciter is located at one side only. Such a one-sided magnetic exciter for vibration excitation of structures and rotors has already been developed and reported [4,5]. Unfortunately a one-sided exciter is nonlinear and even for small displacement of the structure/rotor there is stiffness coupling between the actuator and structure/rotor. A part of the stiffness coupling that varies periodically with time may also be a source of instability.

The present work analyses a hybrid magnetic exciter, which is one-sided, linear and free from mass and stiffness coupling and intended for harmonic excitation of a structure/rotor. It is called a hybrid actuator since it combines a piezoelectric stack actuator and a magnetic actuator. For simplicity in analysis, a single degree of freedom model (SDOF) of a structure is considered for analysis in this paper. If the gap between the vibrating structure/rotor and the exciter can be kept approximately constant, the non-linearity in force due to motion of the structure/rotor is removed. For this, the electromagnet of the exciter needs to be placed on a moving base. Since commercially available piezoelectric stacks are known for their fine positioning capabilities, they are an obvious choice for this purpose. The displacement of the piezoelectric stack can be made almost equal to that of the vibrating structure/rotor using a simple proportional-integral-derivative (PID) control technique.

Since the non-linear effects manifest more in lightly damped structures/rotors, the proposed exciter can be successfully used for such cases. These hybrid exciters can apply forces from a very small value to a moderately high one and work within a frequency of several hundred Hertz. The proposed controller can nicely handle moderate inertia effect of the piezoelectric stack. For very large excitation force and substantial inertia effect, proper tracking control of the hysteretic system may demand for a more sophisticated control strategy.

\section{Analysis}

\subsection{A magnetic actuator with a harmonic current}

It is well known $[1,2,6]$ that the force exerted on a structure/rotor by a one-sided actuator is directly proportional to the square of the current in the electromagnet and inversely to the square of gap between the actuator and the structure/rotor. If the force exerted on the structure is denoted by $F$ (Fig. 1), then

$$
F=k^{\prime} \frac{i^{2}}{\left(x_{0}-x\right)^{2}}
$$

Where $k^{\prime}$ is a constant depending on the geometry, number of turns etc. of the electromagnet, $x_{0}$ is the initial gap between the structure/rotor and the magnet and $x$ denotes the displacement of the vibrating structure/rotor. The displacement $x$ of the structure/rotor is measured from the initial configuration.

When a sinusoidal current $i=I \sin \omega t$ passes through the coil of the electromagnet then the above force in Eq. (1) becomes 


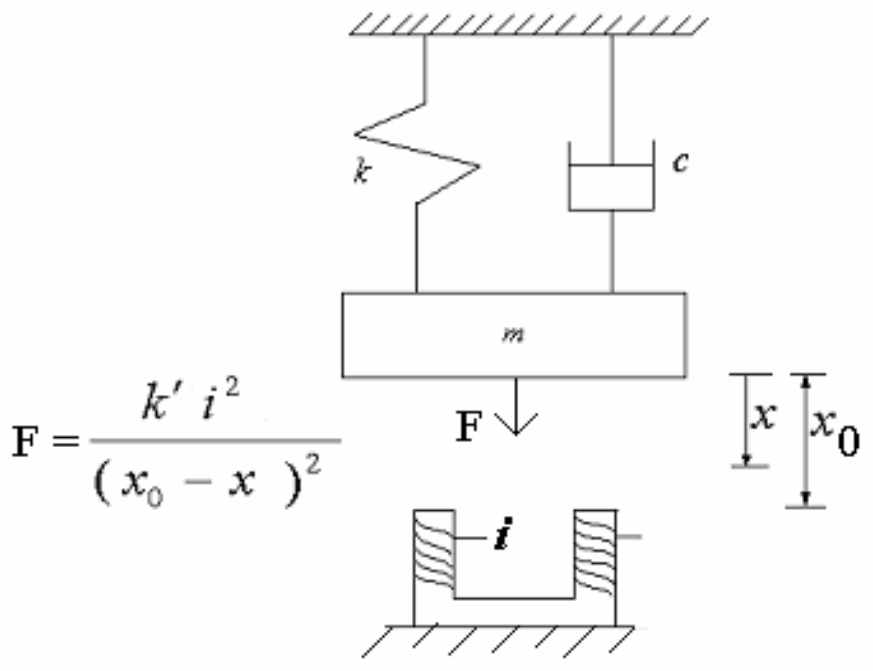

Fig. 1. A non-linear one-sided magnetic actuator.

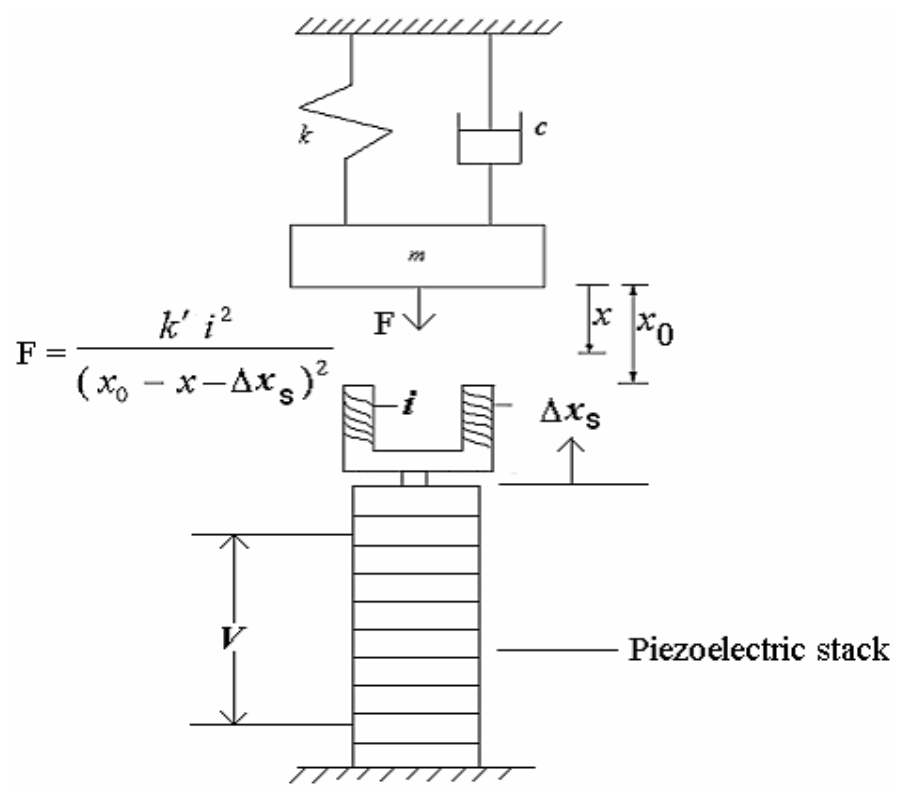

Fig. 2. A one-sided magnetic actuator on a piezoelectric stack.

$$
F=k^{\prime} \frac{I^{2}(1-\cos 2 \omega t)}{2\left(x_{0}-x\right)^{2}}
$$

Therefore, for a one-sided magnetic exciter with harmonic current, the origin of the non-linearity is in the denominator of the expression in the right hand side of Eq. (2).

If the one-sided magnetic exciter is placed on a piezoelectric stack and the increase in length of the piezoelectric stack is denoted by $\Delta x_{s}$ then from Fig. 2 .

$$
F=k^{\prime} \frac{I^{2}(1-\cos 2 \omega t)}{2\left(x_{0}-x-\Delta x_{s}\right)^{2}}
$$


A displacement sensor picks up the displacement of the structure/rotor at the location of the exciter. Then an appropriate control technique makes the motion of the stack $\Delta x_{s}$ approximately equal (with opposite sign) to that of the structure/rotor, i.e., $x \approx-\Delta x_{s}$. The force now becomes

$$
F \approx k^{\prime} \frac{I^{2}(1-\cos 2 \omega t)}{2 x_{0}^{2}}
$$

Thus the denominator is no longer a function of the displacement of the structure/rotor. The force exerted on the structure/rotor does not any more depend on the motion of the structure. However, the frequency of the harmonic part of the force becomes twice of that of the excitation current.

\subsection{Modeling of a piezoelectric stack as a linear one-dimensional element}

Piezoelectric materials are used to convert electrical energy to mechanical energy and vice versa. The precise motion that results when an electric potential is applied to a piezoelectric material is of great practical interest in fine positioning (also called nano-positioning) applications [7,8]. Piezoelectric materials are commercially available in different shapes and sizes. Stack is one of the most common shapes, which can undergo a free axial travel up to $200 \mu m$.

Let $x^{e}(\xi)$ be the displacement along the length of the piezoelectric stack. The symbol $\xi$ is used to represent length along the stack. The bottom end of the stack is fixed and let the displacement at the other end be $x_{s}$. Therefore,

$$
x^{e}(\xi, t)=\frac{x_{s}(t)}{l_{s}} \xi
$$

When the stack is at rest the total length of the stack is denoted by $l_{s}$.

The potential energy expression for the stack [9] is

$$
U=\int_{\xi=0}^{l} \frac{1}{2} \varepsilon \sigma A d \xi-\bar{F} x_{s}
$$

The force on the stack at its free end is denoted by $\bar{F}$. The symbols $\sigma$ and $A$ stand for the stress in the stack and cross-sectional area of the stack respectively. The strain in the stack corresponding to the displacement $x^{e}$ is represented by $\varepsilon=\frac{\partial x^{e}}{\partial \xi}$.

For an applied voltage across the stack, the constitutive relation for the stack [10] is

$$
\frac{\sigma}{E}=\varepsilon-d_{33} \frac{V}{l}
$$

Where the length of a single layer in the stack is $\bar{l}=\frac{l_{s}}{n}$, the voltage applied to the actuator is $V$ and the number of layers in the stack is $n$.

Using Eq. (7) and the relation $\varepsilon=\frac{\partial x^{e}}{\partial \xi}$, the expression (6) becomes

$$
U=\int_{\xi=0}^{l_{s}} \frac{1}{2} \varepsilon \sigma A d \xi=\int_{\xi=0}^{l_{s}} \frac{x_{s}}{l_{s}} A E\left(\frac{x_{s}}{l_{s}}-d_{33} \frac{V}{l}\right) d \xi-\bar{F} x_{s}=x_{s} \frac{A E}{l_{s}} x_{s}-x_{s} \frac{A E}{l_{s}} d_{33} n V-\bar{F} x_{s}
$$

The kinetic co-energy of the actuator is

$$
T=\int_{\xi=0}^{l_{s}} \frac{1}{2} \rho\left(\dot{x}^{e}\right)^{2} A d \xi=\frac{1}{2} \dot{x}_{s} \frac{\rho A l_{s}}{3} \dot{x}_{s}=\frac{1}{2} \dot{x}_{s} \frac{m_{s}}{3} \dot{x}_{s}
$$

The density of the actuator material is $\rho$ and the total mass of the stack is $m_{s}$.

From Eqs (8) and (9), using Hamilton's principle one derives the following equation of motion for the actuator:

$$
\frac{\partial}{\partial t}\left(\frac{\partial(T-U)}{\partial \dot{x}_{s}}\right)-\frac{\partial(T-U)}{\partial x_{s}}=0
$$


Or,

$$
\frac{m_{s}}{3} \ddot{x}_{s}+\frac{A E}{l_{s}} x_{s}=\frac{A E}{l_{s}} \alpha V+\bar{F}
$$

Where, the symbol $\alpha=n d_{33}$ denotes free extension of the stack per unit voltage. Stack actuator extends under a given applied voltage. However, if it is operated around a constant positive voltage $V_{0}$ within its range of operation, it can both extend and contract around that constant position. Any contraction now is actually a contraction superposed on an extension, so that the net change in length is an extension

$$
\frac{m_{s}}{3} \ddot{x}_{s}+\frac{A E}{l_{s}} x_{s}=\frac{A E}{l_{s}} \alpha V_{0}+\frac{A E}{l_{s}} \alpha \Delta V+\bar{F}
$$

The total voltage $V=V_{0}+\Delta V$.

If the above constant position is denoted by the symbol $x_{s 0}$, then

$$
x_{s}=x_{s 0}+\Delta x_{s}
$$

As, $x_{s 0}=\alpha V_{0}$, using relation Eq. (12) in Eq. (11) one gets

$$
\frac{m_{s}}{3} \Delta \ddot{x}_{s}+\frac{A E}{l_{s}} \Delta x_{s}=\frac{A E}{l_{s}} \alpha \Delta V+\bar{F}
$$

\subsection{Modeling of a hybrid exciter}

A one-sided magnetic actuator on a piezoelectric stack is shown in Fig. 2. In the present analysis a simple single degree of freedom model of a structure is considered. The force exerted by the magnet on the mass of the spring-mass system is given by

$$
F=k^{\prime} \frac{i^{2}}{\left(x_{0}-x-\Delta x_{s}\right)^{2}}
$$

The equation of motion for the SDOF spring-mass system is now presented below

$$
m \ddot{x}+c \dot{x}+k x=k^{\prime} \frac{i^{2}}{\left(x_{0}-x-\Delta x_{s}\right)^{2}}
$$

As shown in Fig. 3, the electromagnet pulls the structure/rotor with a force $F$. On the other hand the stack pulls the electromagnet with a force is $\bar{F}$. The inertia force accounts for the difference in the values of these two forces. However, damping has been neglected in this model.

$$
\bar{F}=F-m_{M} \Delta \ddot{x}_{s}
$$

Where the mass of the magnet and its holder is denoted by $m_{M}$.

Using relation (16) and (14) in Eq. (13) one gets

$$
\left(m_{M}+\frac{m_{s}}{3}\right) \Delta \ddot{x}_{s}+\frac{A E}{l_{s}} \Delta x_{s}=\frac{A E}{l_{s}} \alpha \Delta V+k^{\prime} \frac{i^{2}}{\left(x_{0}-x-\Delta x_{s}\right)^{2}}
$$

Equation (15) represents the SDOF spring-mass system with magnetic force while Eq. (17) is used to model the magnetic actuator on the piezoelectric stack.

For a specified harmonic current $i=I \sin \omega t$ in the coil of the electromagnet, the magnetic actuator on a piezoelectric stack works as an exciter. As mentioned earlier, in the present work, the displacement of the piezoelectric stack is made almost equal to that of the vibrating mass, i.e., $x=-\Delta x_{s}$. This makes the force on the vibrating mass in the right hand side of Eq. (15) only dependent on the current. This means the denominator $\left(x_{0}-x-\Delta x_{s}\right)^{2}$ of the force $F$ becomes almost constant, i.e., $\left(x_{0}-x-\Delta x_{s}\right)^{2} \approx x_{0}^{2}$. Under this condition the above exciter is termed as a hybrid one. 


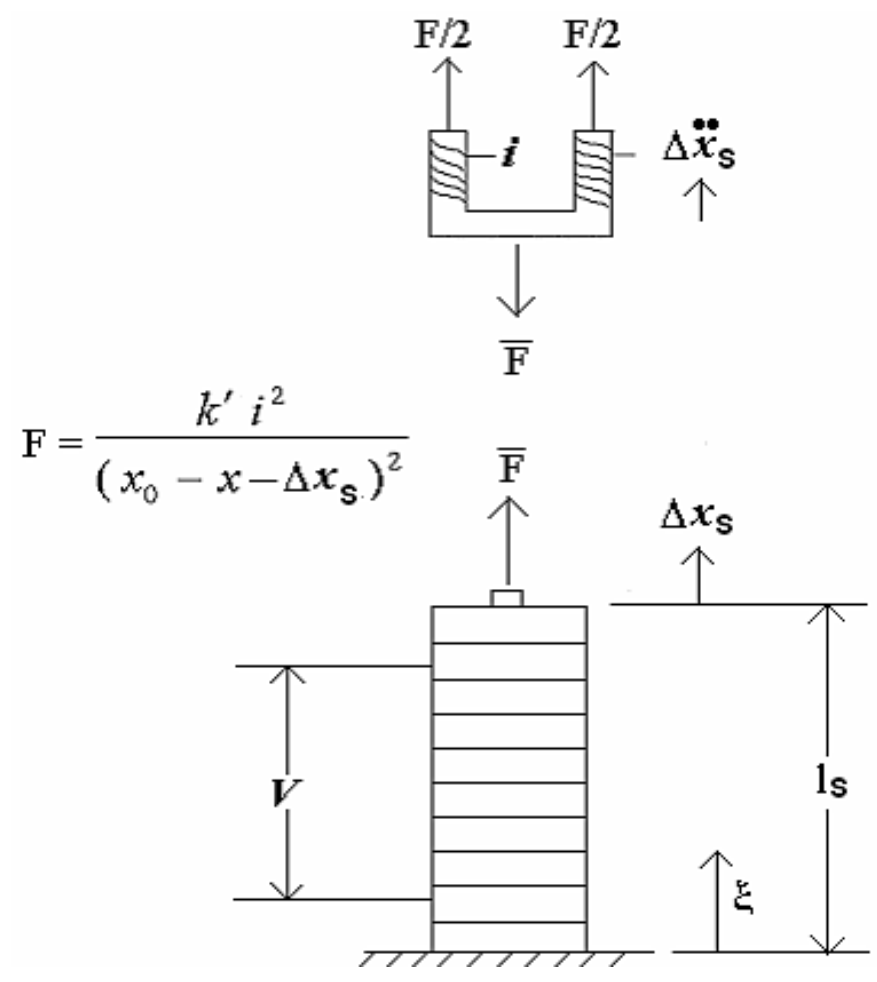

Fig. 3. Forces in the hybrid actuator.

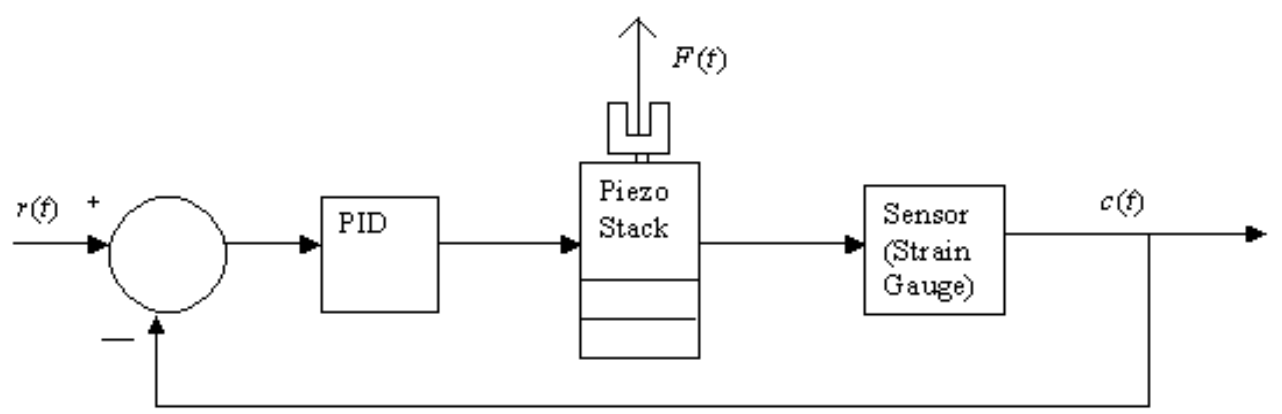

Fig. 4. A controlled piezoelectric stack for positioning.

\subsection{Tracking control in a hybrid exciter}

Equating the vibration of the piezoelectric stack to that of the structure/rotor is a problem of tracking control. The proposed hybrid exciter is to be operated within a frequency of $100 \mathrm{~Hz}$. The piezoelectric stacks generally have natural frequencies of the order of several thousand Hertz. Therefore, for the present case the dynamics of the stack is less important. For this project, the maximum force of the hybrid actuator is limited to $50 \mathrm{~N}$. The combined effect of this force and the weight of the electromagnet with all its fixtures do not have an appreciable effect on the displacement of the stack. Under these conditions the control strategy becomes simple. The control system has to appropriately take care of the hysteresis of the stack. A simple proportional-derivative-integral (PID) control is found to be sufficient for this purpose. A block diagram of the hybrid exciter along with its control system is shown in Fig. 4. Figure 5 shows the working principle of the same. 


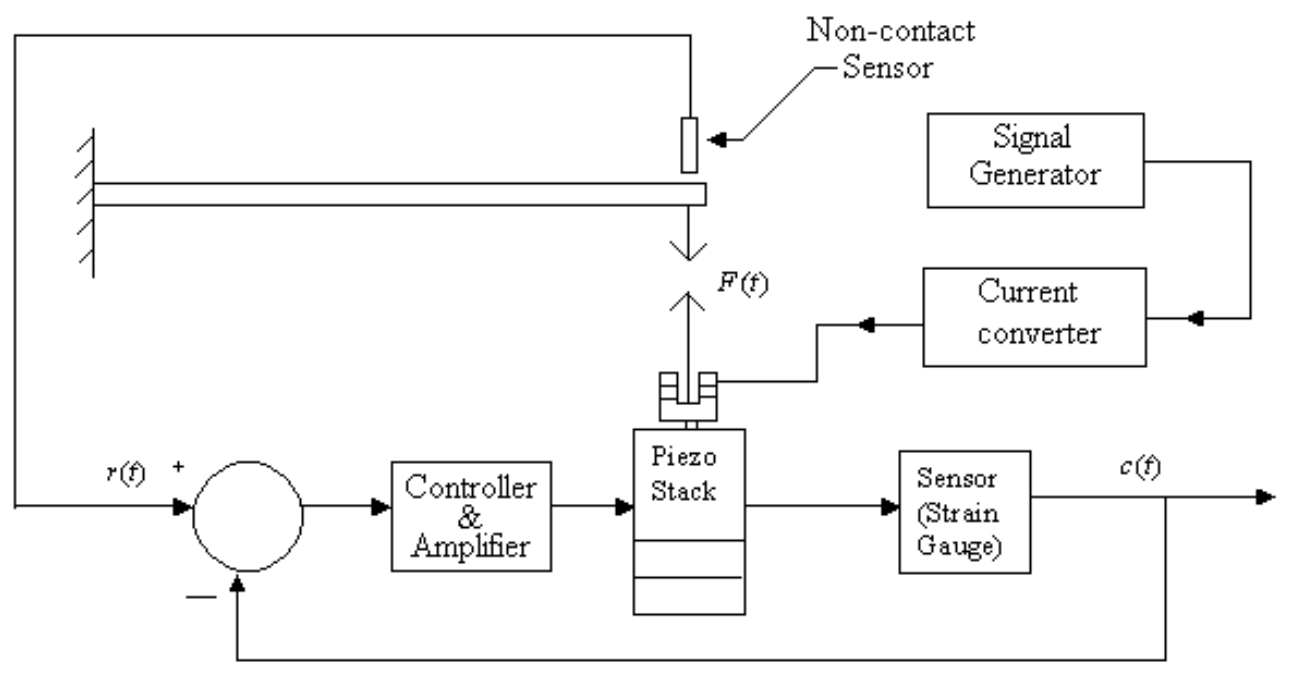

Fig. 5. Schematic diagram showing working principle of the proposed hybrid actuator.

\subsection{SIMULINK model}

The SIMULINK model for a SDOF system with a magnetic actuator on a controlled piezoelectric stack is shown in Fig. 6a and 6b. The subsystem block 'piezo stack with PID controller' shown in Fig. 6a is further expanded in Fig. 6b. In the block 'exciter pos_02' state space representation of a SDOF model of a beam is considered. The shaded blocks are used to generate the force on the structure from the magnetic exciter

$$
F=k^{\prime} \frac{i^{2}}{\left(x_{0}-x-\Delta x_{s}\right)^{2}}
$$

A signal generator, a current converter and a displacement sensor are required for this purpose. A perfect displacement sensor is considered in the SIMULINK model. A filtered value of the displacement of the structure is sent to the controller of the piezoelectric stack. The high pass filter is used mainly to suppress the static (dc) part of the displacement. If the piezoelectric stack is made to extend by this static displacement of the structure, then the dynamic part of the displacement may go beyond the range of its extension. A $3^{r d}$ order analog filter designed based on the Butterworth method is considered for this purpose. A 'gain' block with a gain of -1 is used after the filter because, as shown in Fig. 2, the coordinates $x$ and $\Delta x_{s}$ are opposite in directions. The piezoelectric stack with its controller is shown in Figure 6a as a subsystem. This subsystem (Fig. 6b) accepts filtered displacement data from the displacement sensor. The controller uses this as a reference and makes the piezoelectric stack to follow it. However, the piezoelectric stack with the electromagnet at its free end is approximated as a SDOF system vibrating around its mean position. The following state space representation for the piezoelectric stack is considered here.

$$
\left\{\begin{array}{l}
\Delta \ddot{x}_{s} \\
\Delta x_{s}
\end{array}\right\}=-\left[\begin{array}{ll}
0 & \omega_{n s}^{2} \\
-1 & 0
\end{array}\right]\left\{\begin{array}{l}
\Delta \dot{x}_{s} \\
\Delta x_{s}
\end{array}\right\}+\left\{\begin{array}{l}
1 \\
0
\end{array}\right\}\left\{\omega_{n s}^{2} \alpha \Delta V+\frac{k^{\prime}}{\left(m_{M}+\frac{m_{s}}{3}\right)} \frac{i^{2}}{\left(x_{0}-x-\Delta x_{s}\right)^{2}}\right\}
$$

Where

$$
\omega_{n s}^{2}=\frac{\frac{A E}{l_{s}}}{\left(m_{M}+\frac{m_{s}}{3}\right)}
$$

The symbol $\omega_{n s}$ stands for short-circuited natural frequency of the stack with electromagnet placed on it.

Equation (19) is actually a state space representation of Eq. (17). It has been found that for exciters with small to moderate force ratings, the second term in the right-hand side of the equation has negligible effect in the intended frequency range. 


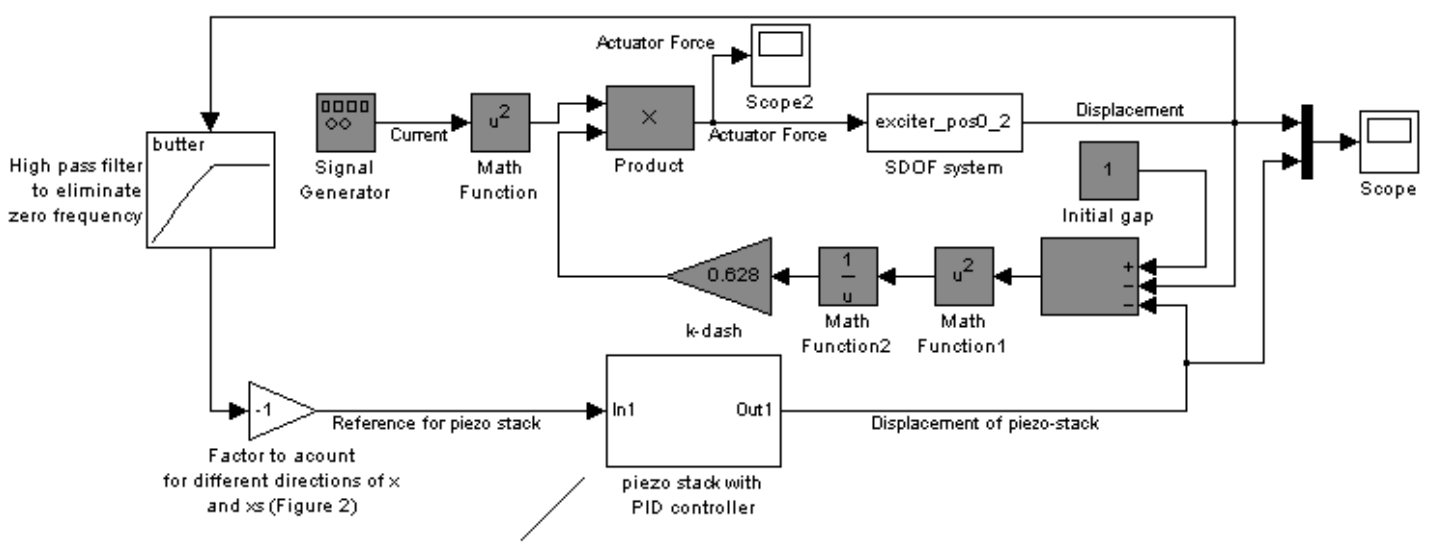

Subsystem expanded in Figure $6 \mathrm{~b}$

(a)

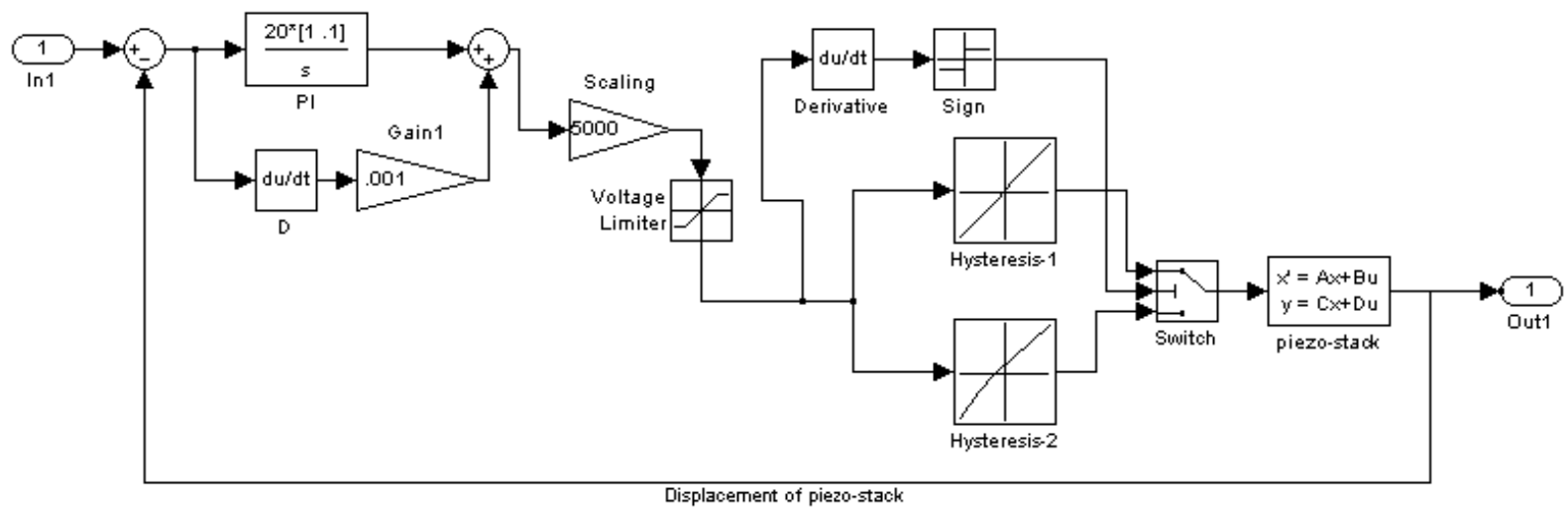

(b)

Fig. 6. (a) SIMULINK model for a single degree of freedom system with the proposed hybrid actuator. (b) The details of the subsystem with the piezoelectric stack and the associated PID control system

The voltage-displacement hysteresis loop is implemented using look-up tables. The displacement versus voltage graph follows two different paths for increasing and decreasing voltages. Though a better model for this hysteresis loop based on the Preisach [11] or the neural network model could have been considered, the simple model used here seems to be sufficient for checking the performance of the controller. If one assumes that the voltage-displacement relation is linear, then the proportional-integral-derivative controller compensates the error due to hysteresis. A voltage limiter is used to protect the piezoelectric stack from voltages beyond its range.

\section{Numerical simulations}

As discussed in the previous section the proposed exciter is used to excite a beam. One end of the beam is fixed and the exciter is placed close to the other one. For the numerical simulation the beam is modeled as a SDOF system and the following data are considered:

The stiffness, mass and damping for the SDOF model of the beam are $k=110 \frac{\mathrm{N}}{\mathrm{mm}}, m=2 \mathrm{~kg}$ and $\xi=0.1 \%$.

For the magnetic actuator the constant $k^{\prime}=0.628 \frac{\mathrm{N} \cdot \mathrm{mm} \mathrm{m}^{2}}{\mathrm{Ampere^{2 }}}$, the mass of the electromagnet with its holder $=m_{M}=$ $2 \mathrm{~kg}$.

For the piezoelectric stack the following properties are considered: 


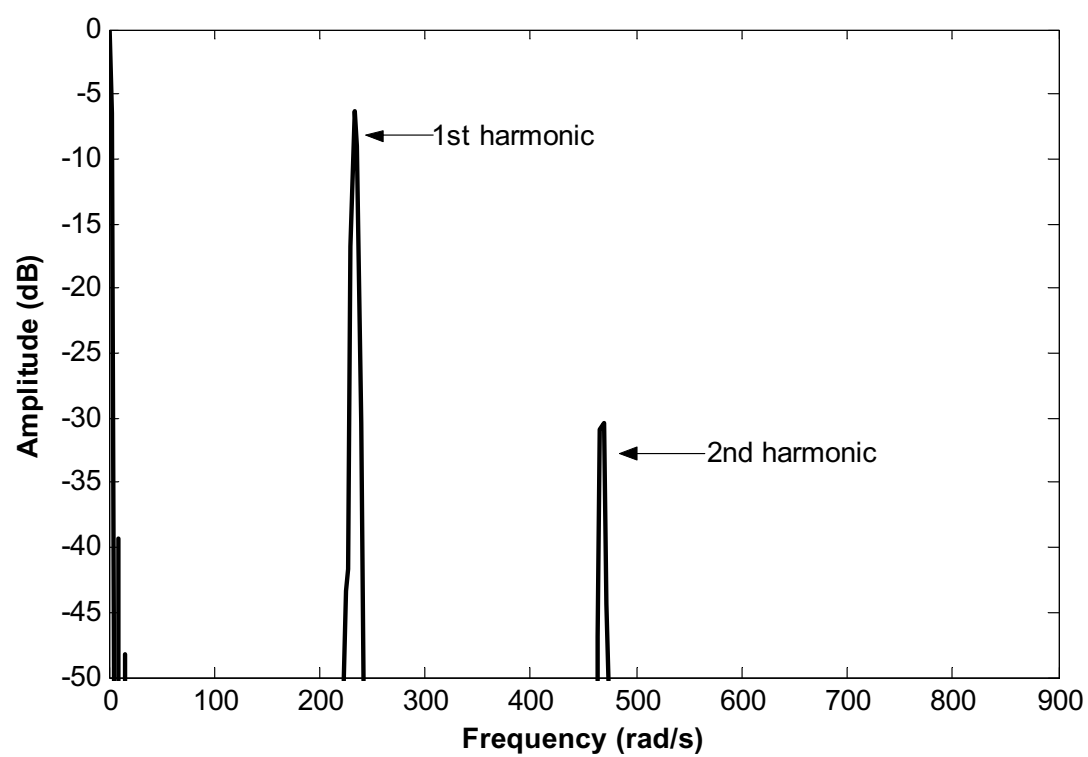

(a)

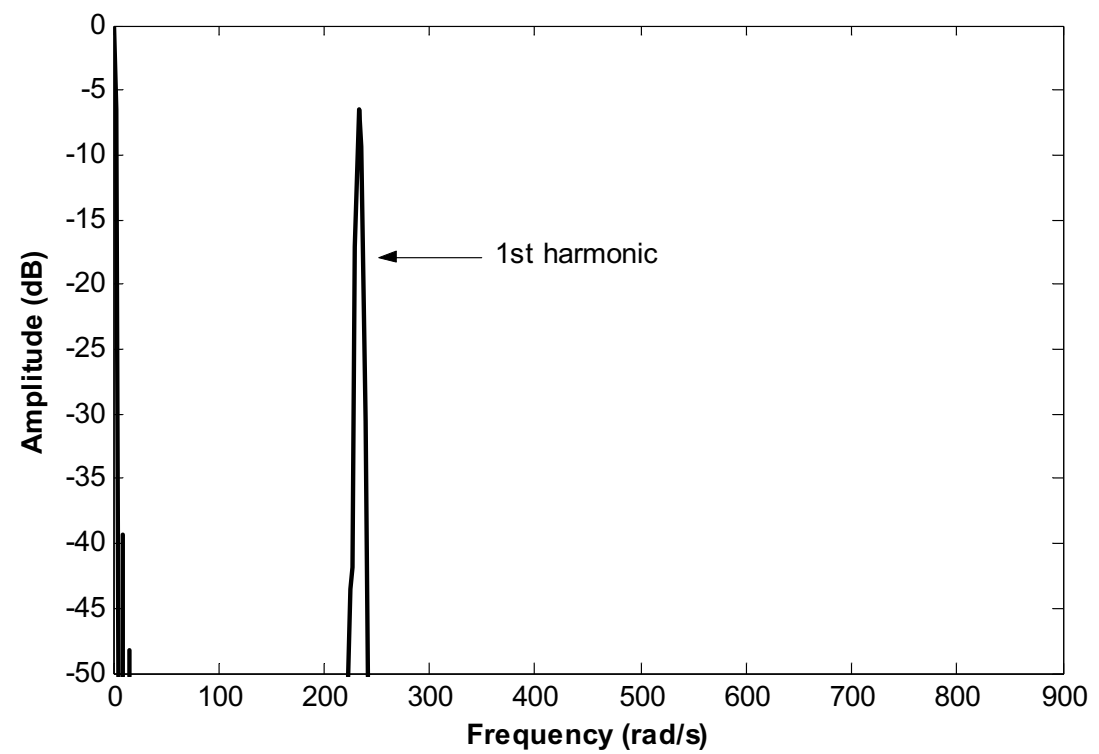

(b)

Fig. 7. (a) The spectrum of force for a one-sided magnetic exciter at an excitation frequency of $2 \omega=234 \mathrm{rad} / \mathrm{s}$. (b) The spectrum of force for the proposed hybrid exciter at an excitation frequency of $2 \omega=234 \mathrm{rad} / \mathrm{s}$.

Open loop stiffness of the piezoelectric stack $=k_{s}=\frac{A E}{l_{a}}=150 \times 10^{3} \mathrm{~N} / \mathrm{mm}$.

Mass of piezoelectric stack alone $=m_{s}=1.8 \mathrm{~kg}$.

Extension of the stack per unit voltage $=\alpha=\frac{1}{5000} \mathrm{~mm} / \mathrm{V}$.

Operating voltage for the stack $=0-1000 \mathrm{~V}$.

Maximum closed loop travel of the stack $=0.2 \mathrm{~mm}$.

The piezoelectric stack operates around a mean voltage of $500 \mathrm{~V}$ and it can travel a maximum displacement of $0.1 \mathrm{~mm}$ in either direction. Therefore, the amplitude of dynamic displacement of the structure should not exceed $0.1 \mathrm{~mm}$. With the electromagnet and its holder the piezoelectric stack has its short-circuited natural frequency at 


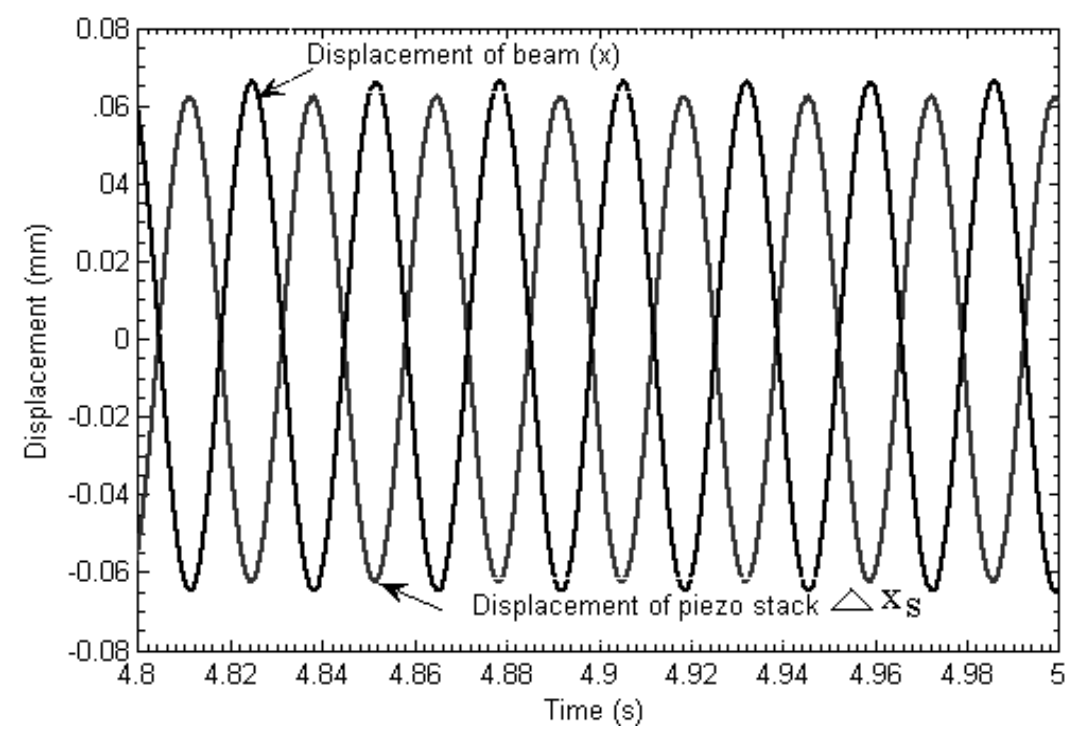

Fig. 8. The displacements of the beam (dc part filtered) and the piezoelectric stack.

$\frac{1}{2 \pi} \sqrt{\frac{k_{s}}{m_{M}+\frac{m_{s}}{3}}}=1209 \mathrm{~Hz}$. The intended range of operation of the exciter is within $100 \mathrm{~Hz}$, which is less than $\frac{1}{10} t h$ of the open-loop natural frequency of the stack. Therefore, dynamic effect of the stack within this operating range is of less importance. For an excitation frequency of $\omega=117 \mathrm{rad} / \mathrm{s}$, the spectrum of the exciter force using an exciter without and with the piezoelectric base is presented in Fig. 7a and Fig. 7b respectively. The presence of the second harmonic (approximately $25 \mathrm{~dB}$ less than the first one) in the spectrum in Fig. 7a shows the non-linearity of the one-sided exciter without the piezoelectric base. The force response of the hybrid exciter shows no second harmonic (in $50 \mathrm{~dB}$ range) in Fig. 7b. Figure 8 shows the displacement $x$ of the beam (dc part filtered out) and that of the stack $\Delta x_{s}$. The difference $x_{0}-x-\Delta x_{s}$ is small and is almost equal to the initial gap $x_{0}$. The small difference in absolute values of the displacements $x$ and $\Delta x_{s}$ is due to the limitation of the proportional-integral-derivative controller in completely eliminating the error arising out of the hysteresis loop in the displacement-voltage characteristics of the piezoelectric stack.

\section{Conclusion}

A simple model of a hybrid one-sided magnetic exciter is performed and its advantage is demonstrated using a numerical simulation. Due to its increased linearity, this hybrid exciter appears to be suitable for vibration excitation of non-linear structures/rotors. As an essential future work the proposed exciters are to be developed for thorough experimental investigations.

\section{References}

[1] A. Chiba, T. Fukao, O. Ichikawa, M. Oshima, M. Takemoto and D. Dorell, 2005, Magnetic Bearings and Bearingless Drives, Newnes, Burlington, MA.

[2] G. Genta, Dynamics of Rotating Systems, Springer, 2004, New York.

[3] H.A. Sodano, Non-contact eddy current excitation method for vibration testing, Experimental Mechanics 46(5) (2006), 627-635.

[4] T. Kreuzinger-Janik and H.Irretier, Experimental Modal Analysis - A Tool for Unbalance Identification of Rotating Machines, International Journal of Rotating Machinery 6(1) (2000), 11-18.

[5] T. Kreuzinger-Janik and H. Irretier, Unbalance Identification of Flexible Rotors based on Experimental Modal Analysis, Proceedings of the International Conference on Vibrations in Rotating Machinery, 12-14 Sep, 2000, Nottingham, UK.

[6] G. Schweitzer, H. Bleuler and A. Traxler, Active Magnetic Bearings, Authors' Reprint, Zurich, 2003. 
[7] S.S. Aphale, B. Bhikhaji and S.O. Reza Moheimani, Minimizing scanning error in piezoelectric stack-actuated nano-positioning platforms, IEEE Transactions on Nanotechnology 7(1) (2008), 79-90.

[8] R.C. Simoes, V. Steffen, Jr., J.D. Hagopian and J. Mahfoud, Modal Active Vibration Control of a Rotor Using Piezoelectric Stack Actuators, Journal of Vibration and Control 13(1) (2007), 45-64.

[9] S.H. Crandall, D.C. Karnopp, E.F. Jr.Kurtz and D.C. Pridmore Brown, Dynamics of Electromechanical Systems, McGraw-Hill, New York, 1968.

[10] A. Preumont, Mechatronics - Dynamics of Electromechanical and Piezoelectric Systems, Springer, Netherlands, 2005.

[11] H. Hu, and R. Ben Mrad, On the Classical Preisach Model for Hysteresis in Piezoceramic Actuators, Mechatronics 13 (2003), 85-94. 

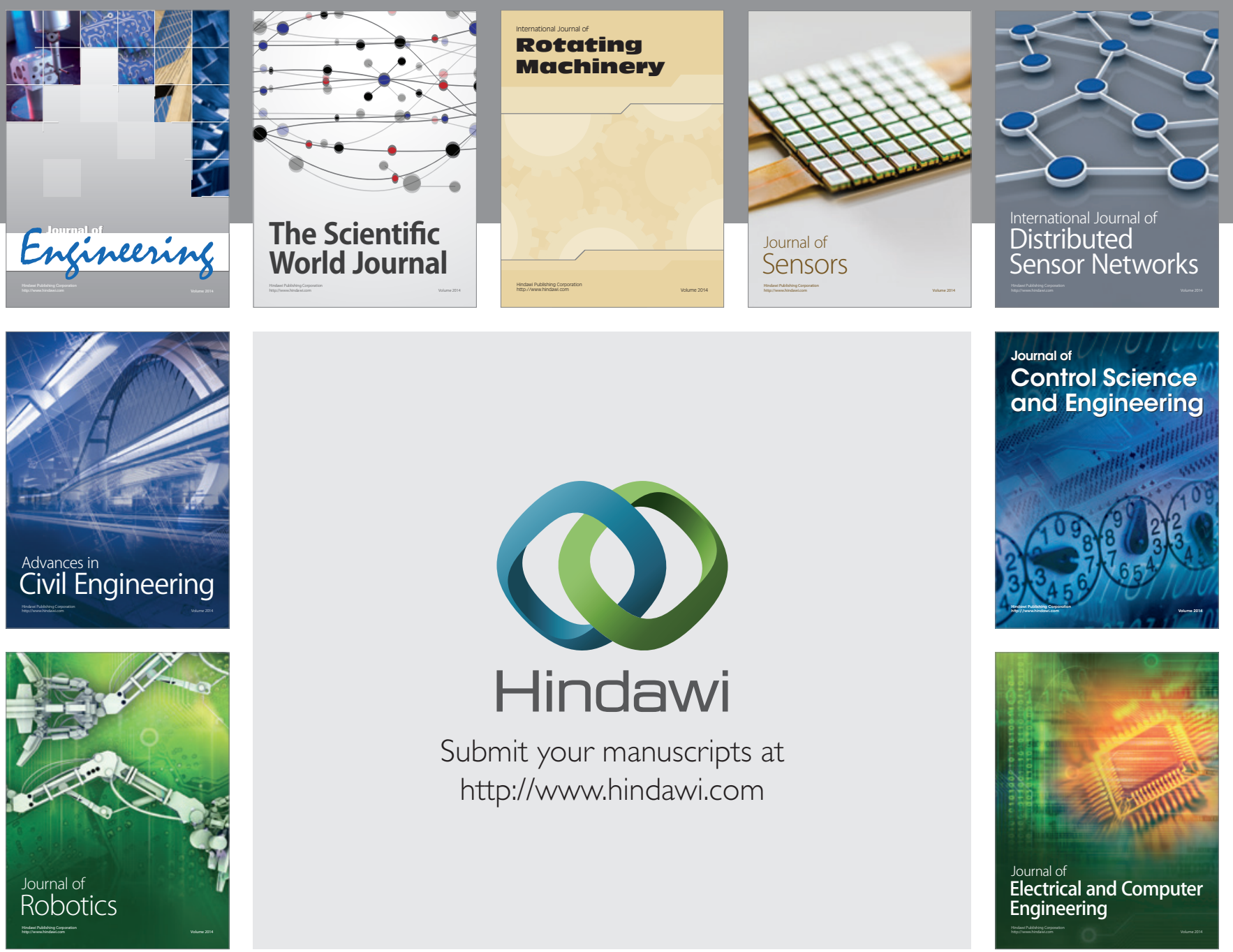

Submit your manuscripts at

http://www.hindawi.com
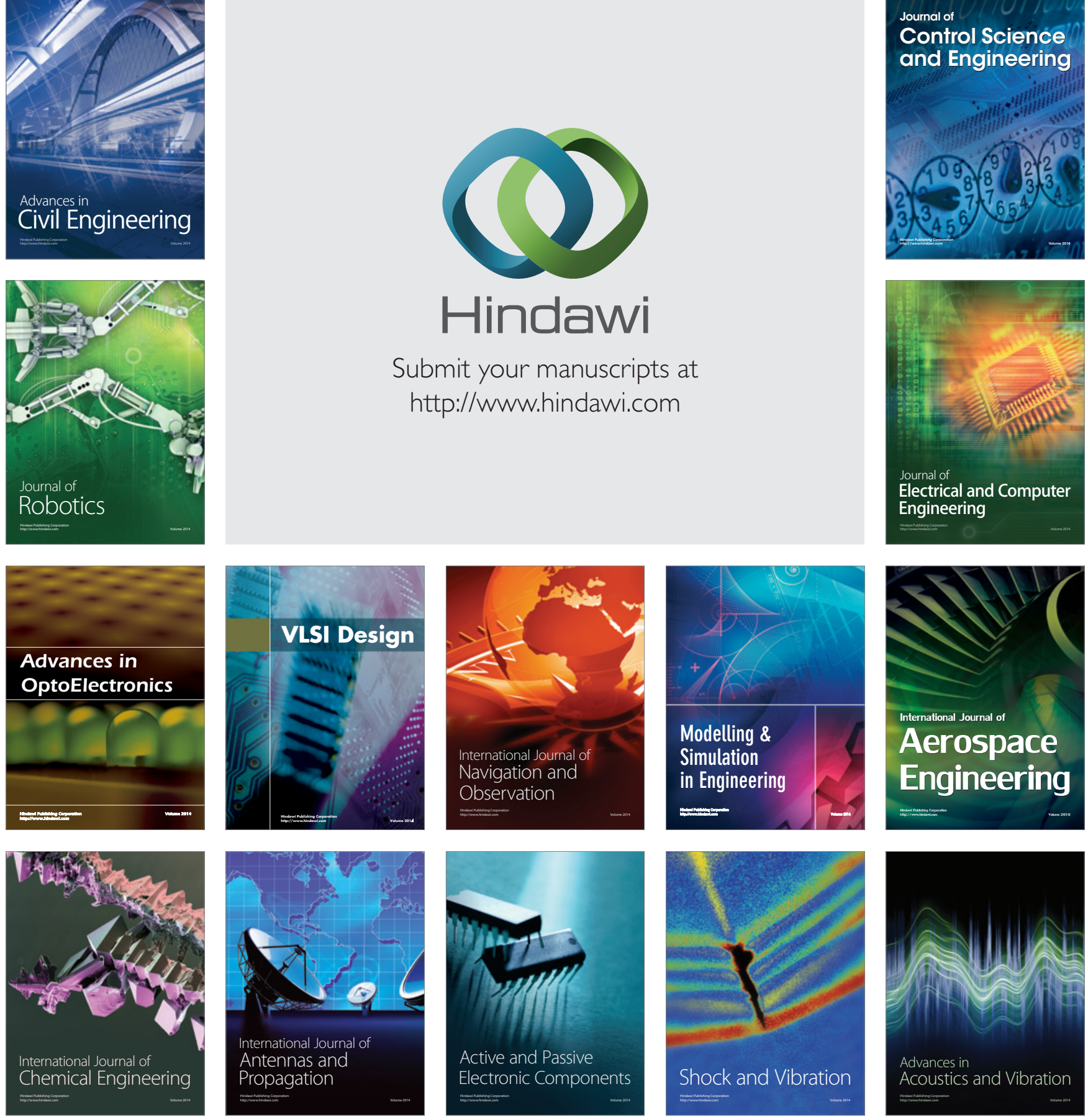